\title{
Single Motor Actuated Peristaltic Wave Generator for a Soft Bodied Worm Robot
}

\author{
Benjamin Winstone ${ }^{1}$, Tony Pipe ${ }^{1}$, Chris Melhuish ${ }^{1}$, \\ Mark Callaway $^{2}$, Appolinaire C. Etoundi ${ }^{1}$, Sanja Dogramadzi ${ }^{1}$
}

\begin{abstract}
This paper presents the design and development of a single motor actuated peristaltic worm robot with three segments using a bio-inspired method of locomotion with one actuator that achieves optimised worm like peristaltic motion. Each segment consists of two solid circular disks that have a tendon connected through to the drive mechanism using a Bowden cable and a soft rubber skin that deforms under the compression of the tendon. Our hypothesis that a tuned peristaltic waveform can achieve improved performance of locomotion distance and clamping strength is proven using an initial test platform capable of demonstrating varying waveform types with multiple actuators. Three experiments were undertaken: (i) moving along a flat surface, (ii) moving through a confined tunnel (iii) moving through a confined tunnel whilst pulling a payload. Results from these experiments have identified the optimal parameters for a smart gearbox capable of achieving the same optimal peristaltic waveform signal as the more complex test platform but with only one actuator driving all three worm segments. Unlike other examples of peristaltic worm robots, this example uses a control method embedded within the mechanics of the design removing excessive number of actuators which contributes to miniaturisation, reduces power consumption and simplifies the overall design.
\end{abstract}

\section{INTRODUCTION}

In the last several decades, many robotics researchers have developed peristaltic locomotion robots [1], [2], [3] and [4]. There are similarities among peristaltic locomotion robots namely drives; DC motors, solenoid actuators, shape memory alloy (SMA), and pneumatic-high frequency drives and miniaturisation is a common goal. From active capsule endoscopy to industrial pipe inspection and space exploration, annelid and more particularly earthworm locomotion demonstrates an effective method of access in to, through and out of hard to reach spaces not easily accessible by humans or more rigid robots. Earthworm locomotion uses waves of muscular contractions along the length of the worms hydrostatic skeleton described as peristaltic locomotion.

Several worm like platforms have been developed for exploration of these confined spaces. Within the context of medical applications peristaltic locomotion provides an action similar to the muscular contractions within the gastrointestinal (GI) tract. Capsule endoscopy describes an untethered swallowable capsule with self contained microsystem used for visual inspection of the GI tract. Capsule endoscopy is passive in nature so its direction and movement is determined by the muscular action of the GI tract. An

\footnotetext{
${ }^{1}$ Bristol Robotics Laboratory, University of the West of England, Bristol, UK Benjamin.Winstone@brl.ac.uk

${ }^{2}$ Department of Radiology, Bristol Royal Infirmary, Bristol, UK Mark.Callaway@uHBristol.nhs.uk
}

active capsule capable of both maintaining a stall state by resisting the muscular action and also moving through the GI tract to a specific location would greatly open up the potential of technology towards targeted diagnostic and treatment procedures. Efforts have been made towards active capsule endoscopy [5], [6] in particular Zuo et al. [7], Yan et al. [8] and Zarrouk et al. [9] present peristaltic earthworm robots and Gao et al. [10] present an inchworm like locomotion using inflating balloons to achieve clamping segments, however these mechanism use several actuators and complex transmission systems.

Other examples exist of robots designed for more generic applications in mind. Omori et al. [11] present a seven segment worm with two actuators in each segment capable of bidirectional linear movement and cornering. Whilst being a successful example of peristaltic worm locomotion each segment requires two actuators which increases complexity, size, power requirements and control. Towards a more simple design Seok et al. [12] present the meshworm a soft robotic platform that exhibits peristaltic locomotion. This worm robot used micro NiTi coil actuators wrapped around a soft braided body. This robot is mechanically simple and naturally robust through its compliance. It does however require a specific control sequence to achieve locomotion. The work of Boxerbaum et al. [13], [14] [15] presents an effective mechanism that uses one rotary actuator that generates sinusoidal waves to multiple tendons that set at varying phases around the centre of rotation. In combination with a mechanically hinged body that behaves similar to the braided meshworm, this robot exhibits excellent peristaltic locomotion from one actuator. Miniaturisation of this system may present problems when reducing the large number of mechanical joints and cable runs within the body .

In order to develop robots capable of exploration of small spaces in particular within a medical context a simpler mechanical design would aid in miniaturisation and robustness. The examples discussed use multiple actuators or complex bodies that require more complex control signals. Boxerbaum's worm uses a relatively simple drive mechanism however more recently Horchler et al. [16] have further developed the work of Boxerbaum by adding multiple actuators along the length or the body further complicating the control system. A gap has therefore been identified that shows a lack of development towards a worm robot with a simplified actuation sequence driven by only one actuator. The intention of this research is to build on previous work with a focus on simplifying the design so that future miniaturisation 
is possible. Reflecting particularly on the earlier work of Boxerbaum it is felt that a biomimetic tendon contraction sequence can be achieved from one actuator to control a soft bodied robot. This paper investigates the compromises of using only one actuator to drive multiple segment contracts and whether a smart gearbox can be used to fine tune the control of the actuator tendons that contract each segment.

\section{PERISTALTIC WORM LOCOMOTION}

\section{A. Biomimetic inspiration}

The robot locomotion method is based upon the peristaltic motion adopted by annelids, in particular the earthworm. Locomotion is achieved by waves of muscular contractions along the length of the hydrostatic skeleton that shorten and lengthen body segments. Whilst the shortened segment anchors to the environment surface, the lengthened segment advances the body along the trajectory, see Fig. 1. This action is achieved using a combination of circular muscles and longitudinal muscles creating the extension and contraction movements respectively. As a segment contracts longitudinally it will expand creating a increased friction of locking action to the surrounding environment. The clamping action provides the opposing forces to allow the remaining segments to extend longitudinally and advance the body of the worm forwards.

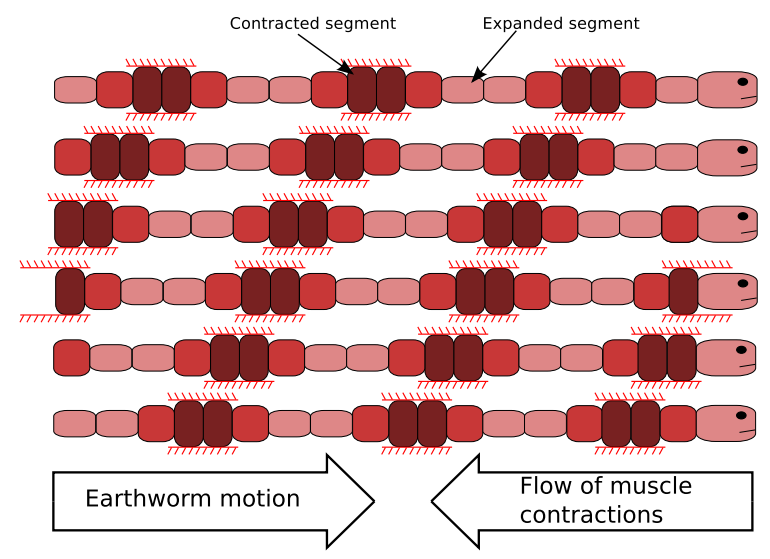

Fig. 1. Earthworm peristaltic locomotion showing waves of muscular contractions.

\section{B. Actuator mechanism}

With the intention of reducing mechanical complexity and the number of actuators a robot design is considered that uses only one motor to contract multiple segments of a peristaltic worm. During forward linear locomotion of an earthworm each individual segment of the body is performing the same action as the other segments, only at a different phase. This suggests that each individual segment could be actuated from the same actuator if the phase of each segment can be offset. The work of Boxerbaum et al. [13] presents an effective mechanism that actuates multiple tendons from one rotary actuator. Using bowden cables rotational movement of the motor can be translated in to linear movement or contraction of a segment. With each bowden cable positioned at a phase offset the corresponding worm segment will contract at a unique period in the motor's cycle. Omori et al. [11] state that a minimum of three worm segments are required to achieve peristaltic motion so from this point on we will consider a robot of three segments. Fig. 2 demonstrates the principles of this mechanism with three segments. An axially positioned motor rotates a free moving cam housing which all tendons attach to. As the cam rotates it pulls an individual tendon by different displacements based on its angular position or phase offset. The amount of displacements of a tendon at any given time relates to the angle of the motor and phase offset of the tendon, see equation 1. As shown in Fig. 2 the output of this mechanism to each segment is a sinusoidal wave through the contracting tendon.

$$
h=\sqrt{(t x-(r 1 \cdot \cos (\theta)))^{2}+(t y-(r 1 \cdot \sin (\theta)))^{2}}
$$

Where;

$r 1=$ Distance from motor shaft centre to spur gear centre

$t x=$ Tendon exit point horizontal distance from centre

ty $=$ Tendon exit point vertical distance from centre

$h=$ Distance the tendon is pulled at angle $\theta$

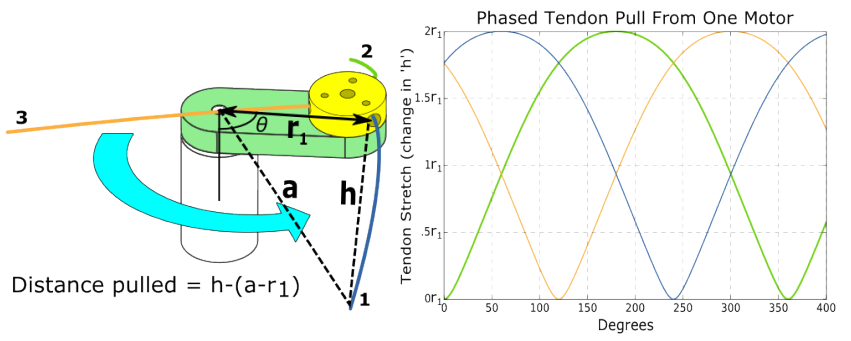

Fig. 2. Phased linear tendon pull with single rotary actuator.

\section{State driven actuator mechanism}

Earthworm locomotion is based on waves of muscular contractions traveling the length of the body. However, It is not a fluid motion of each segment being in constant transition. Segments that clamp or root to the environment hold their state for the full length of time in which the remaining segments complete their transition to either extension or contraction, see State Table. I. As such the sinusoidal control of segment tendons is not sufficient to achieve such behaviour. Instead, a waveform that contains three stages; Contract, Extend and Clamp is required. Whilst a rotational motor output can directly translate to a sinusoidal movement, a more complex mechanism is needed to achieve this new waveform.

Omori et al. [11] state that at least three segments are required to achieve peristaltic motion. Three worm segments are considered, one for each of the described states. As the swing arm rotates between the contracting and extended 
TABLE I

Three Segment Worm Locoomotion State Table

\begin{tabular}{|c|c|c|c|}
\hline State & Segment 1 & Segment 2 & Segment 3 \\
\hline $\mathrm{t}_{0}$ & Clamp & Contract & Extend \\
\hline $\mathrm{t}_{1}$ & Extend & Clamp & Contract \\
\hline $\mathrm{t}_{2}$ & Contract & Extend & Clamp \\
\hline $\mathrm{t}_{3}$ & Clamp & Contract & Extend \\
\hline $\mathrm{t}_{4}$ & Extend & Clamp & Contract \\
\hline
\end{tabular}

tendon points, the pull on the clamping segment must remain the same length throughout the duration of the transition. This determines the loci of the output of the mechanism as a three sided shape with each side having a degree of curvature. In order to achieve such a loci with a specific number of sides, a number of examples of working systems can be considered; e.g. the geometric drawing toy Spirograph demonstrates how two counter rotating wheels can produce multi-sided shapes.

\section{The Effect of Radii Ratio on Resulting Loci Path}
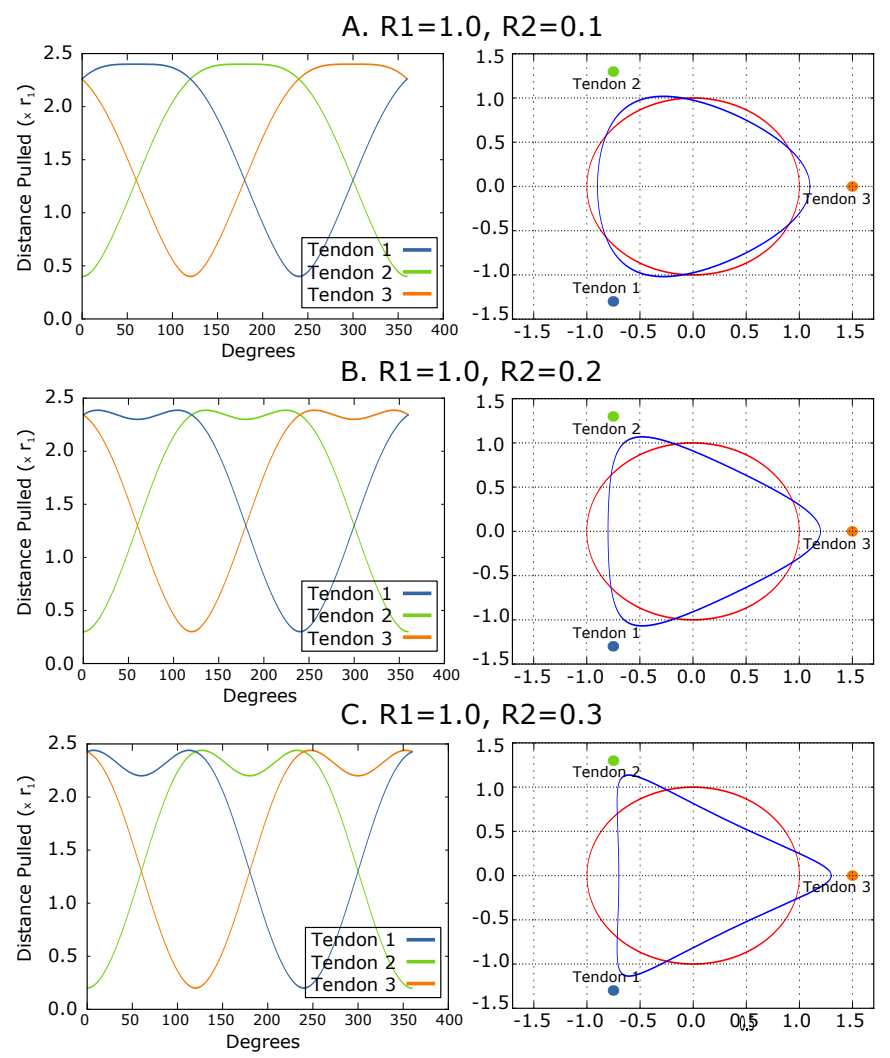

Fig. 3. The left column of graphs show the tendon pull or waveform against angular position of the motor, the right column of graphs show the loci generated. The loci path produced by the gear mechanism changes according to the ratio between the two radii. A longer period of flat or most tedon tension signified in A. shows more effective segment clamping than in $\mathrm{C}$. where clamping relaxes mid way through the clamping stage.

The two counter rotating wheels that generate a triangular loci from an outer point of the second wheel generate the waveform and shape produced in Fig. 3. By varying the ratio between the radius of the first and second wheel the waveform can be varied to achieve either a longer but more unstable clamping phase or a shorter but more stable clamping phase. To achieve the most stable clamping phase where the tendon retains stationary tension throughout, the distance of the loci point to the tendon attachment point must remain the same during the clamping phase. During this period the remaining two tendons either undergo a pulling or releasing transition. By translating this triangular loci to linear movement of the tendons that contract and radially expand the worm segments, a bio-mimetic locomotion method can be achieved.

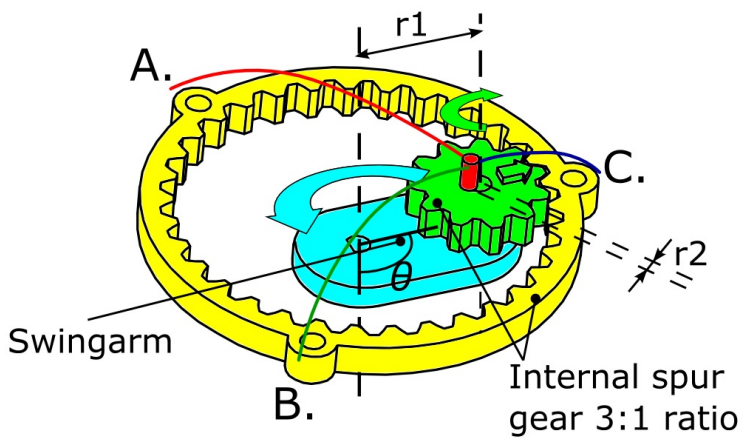

Fig. 4. Diagram of the planetary gear arrangement needed to achieve the correct waveform.

The gear arrangement is designed as shown in Fig. 4 where r1 shows the distance from the motor shaft to the spur gear and $\mathrm{r} 2$ is the distance from the central axis of the gear to the offset point to which the tendons $\mathrm{A}, \mathrm{B}$ and $\mathrm{C}$ are attached to. In order to determine how much a tendon is pulled at any given motor position, a simple calculation can be applied to sum the vectors of the two gear radii to achieve an $\mathrm{x}, \mathrm{y}$ position and then calculate the distance to the tendon exit point.

$$
\begin{gathered}
x=(r 1 \cdot \cos (\theta))+(r 2 \cdot \cos (-2 \cdot \theta)) \\
y=(r 1 \cdot \sin (\theta))+(r 2 \cdot \sin (-2 \cdot \theta)) \\
h=\sqrt{(t x-x)^{2}+(t y-y)^{2}}
\end{gathered}
$$

Where;

$x=\mathrm{X}$ coordinate of tendon attachment wheel

$y=\mathrm{Y}$ coordinate of tendon attachment wheel

$r 1=$ Distance from motor shaft centre to spur gear centre

$r 2=$ Distance from spur gear centre to tendon wheel offset

$t x=$ Tendon exit point horizontal distance from centre

ty = Tendon exit point vertical distance from centre

$h=$ Distance the tendon is pulled at angle $\theta$

\section{TUNING PARAMTERS}

In the following analysis, it is assumed that the worm segments radial expansion will make contact a with an outside surface when its axial contraction reaches 1.8 times 
$r_{1}$. The maximum a rotating swing arm of length $r_{1}$ can pull a tendon is $2 r_{1}$. This allows further expansion for the flexible skin to comply and obtain a footing with the surface.This paper focuses on identifying gearbox parameters that are tuned for the environmental size, such that the worm could compensate for a smaller or larger pipe and obtain stronger clamping force.

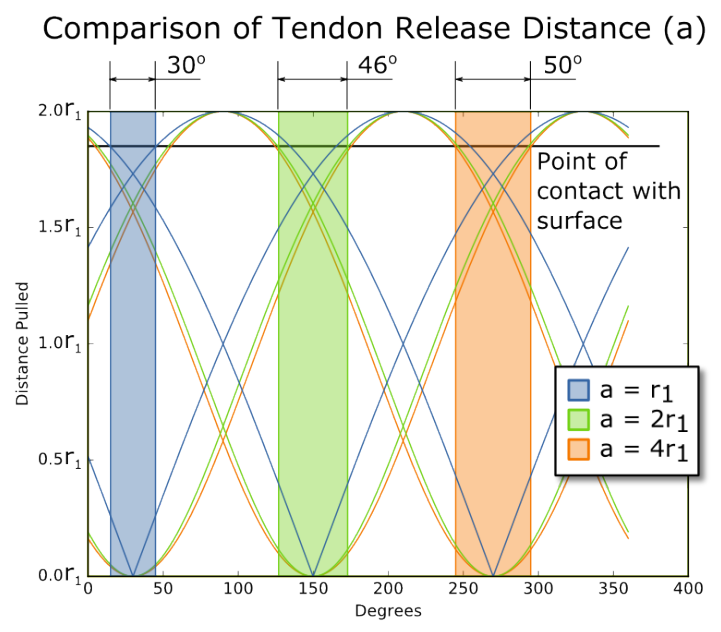

Fig. 5. Effect of changing tendon release point, a, in a basic swingarm with no gearbox. Shaded regions detail periods of no clamping so no effected movement.

\section{A. Tendon offset}

A number of factors affect the performance and efficiency of this design. Consider a system that uses a swing arm of length $r_{1}$ attached to a motor. A tendon release point is placed at distance ' $a$ ' from the centre of rotation. The distance from the tendon release point to the end of the swing arm, $h$, will vary through the rotation of the motor as shown in Fig. 2. Although a rotation can translate to a sinusoidal waveform, the distance of the tendon release point to the centre of rotation affects the conformance to an exact sinusoidal form. Fig. 5 demonstrates the effect of changing length $a$ from a distance of $r_{1}$ to $4 r_{1}$. This shows that a tendon positioned closer to the point of rotation produces a sharper trough shape, see Fig. 5. In the context of achieving the perfect waveform for effective peristaltic worm locomotion, attention should be focused on maximising the period in which the wave is above the point of contact so that the least amount of time is spent transitioning between phases whilst having no contact with the surface. Fig. 5 shows shaded regions that relate to each configuration of length $a$, highlighting the crossover point with the contact threshold. Essentially the wider the shaded region the greater the time the configuration is not clamping during a transition. This shows that a tendon release point at a distance of $r_{1}$ from the centre of rotation achieves clamping for the longest period, $90^{\circ} / 120^{\circ}$ compared to the furthest distance of $4 r_{1}$, $70^{\circ} / 120^{\circ}$.

We consider a gearbox mechanism driven by this previous example of a swing arm of length $r_{1}$ attached to a motor.
The gearbox enables a counter rotating gear at a ratio $3: 1$ that connects to the free rotating pulley wheel that connects to the tendons as shown in Fig. 4. As with previous examples of varying the tendon release point 'a', the effect of using the gearbox mechanism is the same, however the ineffective periods are greatly reduced to $9^{\circ} / 120^{\circ}$ when the distance is $r_{1}$, and $61^{\circ} / 120^{\circ}$ when the distance is $4 r_{1}$, see Fig. 6.a.

\section{B. r1:r2 ratio}

The main attributing variable of the gearbox is the ratio between length $\mathrm{r} 1$ and $\mathrm{r} 2$ as shown in Fig. 4. The effect of changing this ratio is shown in Fig. 6.b where an increased $\mathrm{r} 2$ in relation to $\mathrm{r} 1$ increases the period of time over the clamping threshold. However, a further increase of $r_{2}$ creates a dip at the peak of the wave which can cause the wave to drop below the contact threshold momentarily compromising contact with all sides of the robot. If the contact threshold is $1.8 r_{1}$ the ideal ratio between $\mathrm{r} 1$ and $\mathrm{r} 2$ is 1:0.15 where the period of clamping is increased to $114^{\circ} / 120^{\circ}$.

\section{Effeciency improvement of gearbox}

When a worm segment contracts, it clamps to the contact surface for the entirety of its cycle, allowing the remaining segments to advance axially. At any given time, one of the segments is clamped to the contact surface, achieving an overall stronger and more effective clamping. A waveform for such a situation is shown in Fig. 7 noted as an ideal waveform. A sine waveform generated by the simple rotating swing arm pulling offset tendons is shown with no gearbox, and finally with the gearbox waveform. Highlighted are the periods of no clamping when the contact surface is engaged at an axial compression of 1.8 times $r_{1}$. The parameters for the gearbox waveform use the $r_{1}: r_{2}$ ratio of 1:0.15 and the tendon offset of $1.2 r_{1}$ to allow for the addition of $r_{1}$ to $r_{2}$ at the peak of the waveform. Fig. 7 shows that whilst an ideal waveform achieves $100 \%$ clamping per cycle, the gearbox derived waveform only suffers a $6^{\circ}$ or $5 \%$ sacrifice. However, a simpler swing arm generated sine waveform that suffers a $23^{\circ}$ or $19.2 \%$ sacrifice in clamping per cycle.

\section{ROBOT DESIGN}

\section{A. Gearbox design test platform}

Before designing the gearbox mechanism proposed in this paper, the waveforms suggested must be compared. This has been achieved through building a robot worm body with actuators in each segment, as shown in Fig. 8. The robot worm has been printed on an Objet 260 3D rubber printer where flexible worm segment skins are made of Tango Plus, and the rigid sections, Vero White. Each worm segment comprises of 4 rubber segment skins. These skins expand radially when contracted axially. A Dynamixel XL320 motor and pulley connect both ends of the segment with a tendon cable. When the segment expands radially it gains friction with the supporting or contacting surfaces. 
a) Varying Tendon Offset

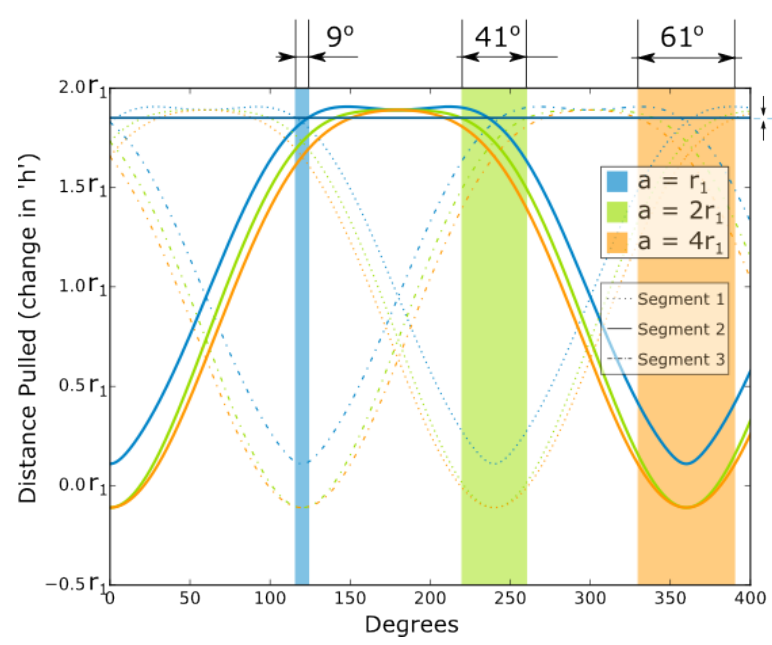

b) Varying $r_{1}: r_{2}$ Ratio

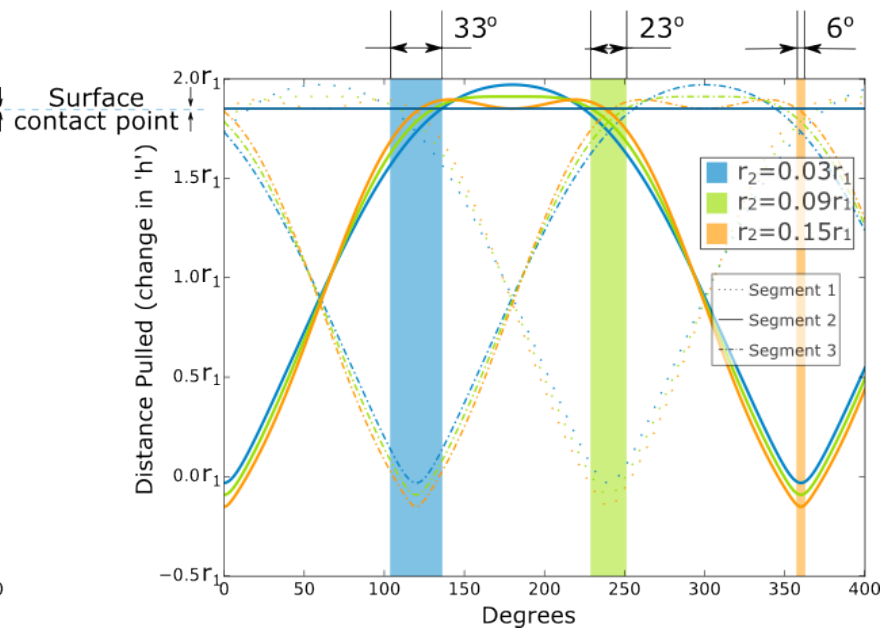

Fig. 6. (a) Comparison of effective clamping period when varying tendon offset in gearbox mechanism, where $r_{2}=0.09$. (a) Comparison of effective clamping period when varying $r_{1}: r_{2}$ ratio in gearbox mechanism, where $a=1.2 r_{1}$.

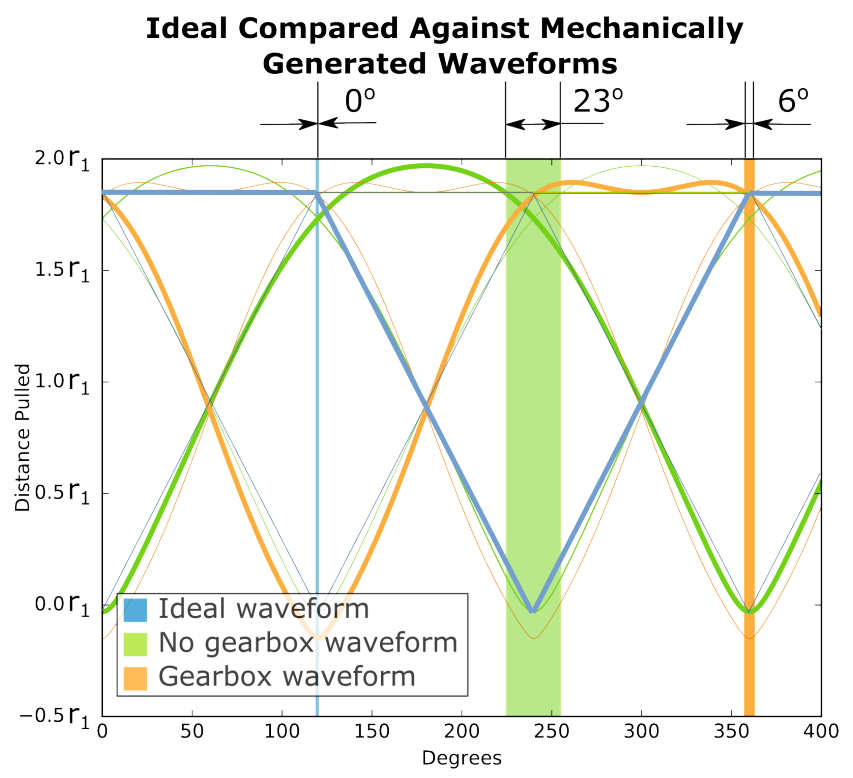

Fig. 7. Comparing an ideal waveform, sine wave swingarm generated waveform an optimised gearbox generated waveform.

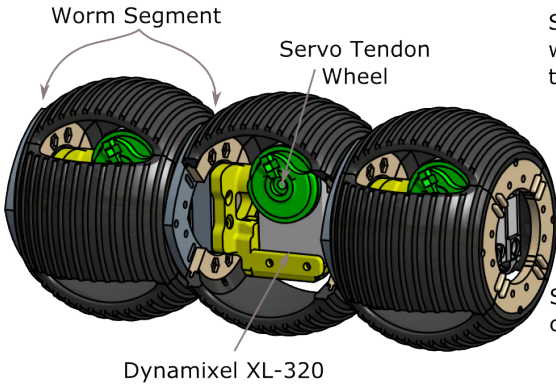

a) Worm Assembly
Servo pulley wheel rotates to pull tedon

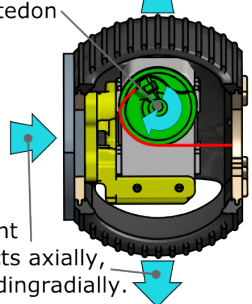

b) Worm Segment
Fig. 8. Dynamixel driven robot built to test out each waveform and compare performance and identify optimal gearbox parameters. a) Assembly of three worm segments, each containing a Dynamixel xl-320 servo. b) One worm segment showing how the dynamixel servo rotation pulls the tendon compressing the segment.
1) Force distribution test: In order to understand the performance of this test platform a relationship between segment contraction and the distributed force needs to the understood. Fig. 10 displays results of load cell measurements of the forces imposed on the contacting surface when the segment is compressed by an axial force. One segment has been compressed from above, whilst the forces applied by each of the segment walls to their contacting surfaces are measured by load cells, as shown in Fig.9. The results show that the forces are distributed equally. The inclusion of the actuator inside the segment limits space for compression of the segment to $80 \%$ of the original axial length. To achieve this axial compression of $20 \% 4 \mathrm{~N}$ of force is required. The radius of the servo pulley is $10 \mathrm{~mm}$ and so the minimum torque of the motor required to achieve this compression is $0.04 \mathrm{Nm}$. The Dynamixel XL-320 achieves $0.4 \mathrm{Nm}$ is a sufficient actuator for the design.

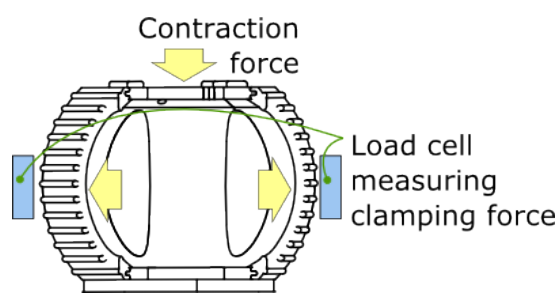

Fig. 9. Force distribution test.

2) Locomotion on a flat surface: The test platform worm robot has been subjected to three tests: 1. locomotion on a flat surface, 2. locomotion within a confined tunnel where all worm's segments contact during the clamping phase and 3 . locomotion whilst pulling a payload. In these experiments, one cycle is considered to be a segment going through all transitions. This corresponds with one full rotation of the swing arm. 50 cycles of the three waveforms are passed to the worm robot and the corresponding distance travelled across 
Force Measured at Contact Surface of Each Worm Segment

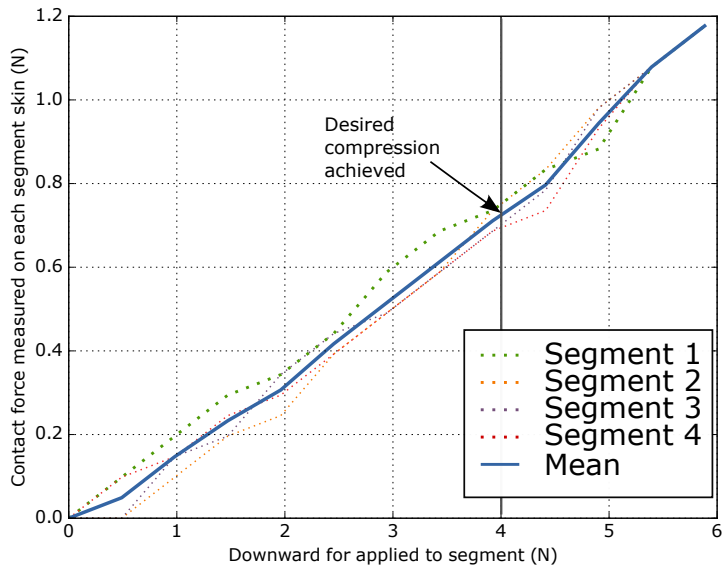

Fig. 10. Force distribution measurement of each segment when subjected to axial compression.

an acrylic surface is measured. $155.0 \mathrm{~mm}$ was achieved by the standard sine waveform at $155.0 \mathrm{~mm}, 173.0 \mathrm{~mm}$ by the generated gearbox waveform and $175.0 \mathrm{~mm}$ by the ideal waveform. In summary the sine waveform is 86.57 $\%$ of the best achieved whilst the gearbox waveform is $98.86 \%$ of the best achieved. This highlights that, on a flat surface a worm with one actuator would sacrifice only $1.13 \%$ drop in performance if the gearbox mechanism is used compared to a simple swing arm mechanism that loses $13.43 \%$. The movement of the tip of the robot's segment skin during contraction is being tracked, see Fig.11. This shows the distance achieved per cycle, and also the fact that the contraction amplitude is higher when the robot travels further.

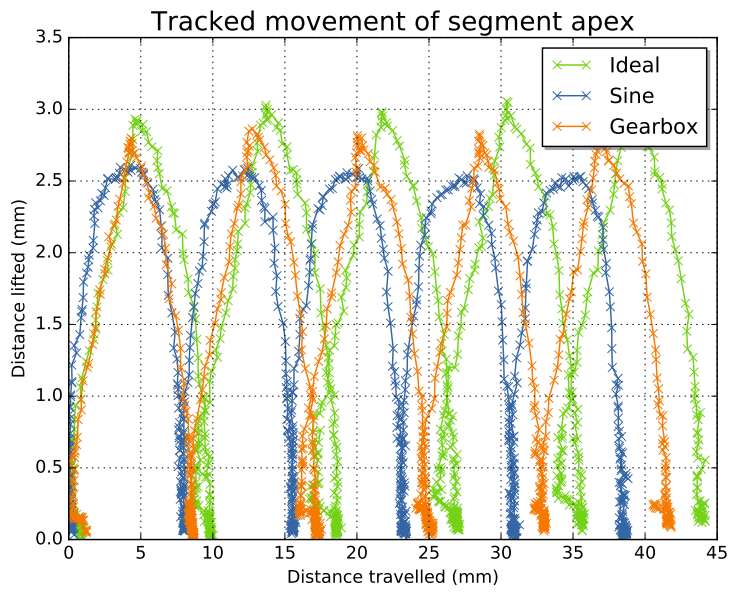

Fig. 11. Tracked segment apex of worm robot travelling across a flat surface.

3) Locomotion through a confined space: A confined tunnel has been constructed with a width equal to the measured expansion of a segment when it is contracted to 1.8 times $r_{1}$, see Fig. 12. Given the limitation of space in the segment allowing for $20 \%$ axial contraction, $9.6 \mathrm{~mm}$, r1 will be considered to be half of this distance, $4.8 \mathrm{~mm}$. When a segment is contraction to 1.8 times $r_{1}$ it expands radially to $76 \mathrm{~mm}$, which determines the width of the tunnel. The first experiment within the tunnel simply measures the distance travelled when all four segment skins contact the surrounding environment. Table II shows the results of this experiment, where the efficiency of the gearbox waveform compared to the two other waveforms is in line with the locomotion on a flat surface. However, in all cases, the distance achieved is greatly reduced due to the resistance incurred from the four segments skins in contact with a surface compared to only one skin surface contacting when on a flat surface.

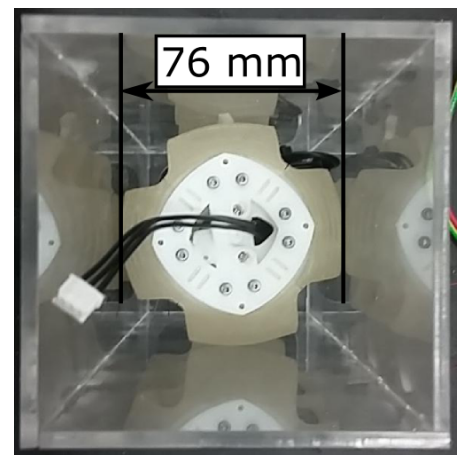

Fig. 12. Front view looking in to tunnel created for confined space experiment. The width of the tunnel is $76 \mathrm{~mm}$ to match the diameter of the worm when a segment is contracted to $1.8 r_{1}$.

4) Locomotion through a confined space with payload: The final experiment investigates how well the worm robot can pull a payload whilst inside the confined space. In this instance brass weights were attached to the back of the worm to be pulled by the robot. The static coeffecient between the acrylic surface and brass weights has been empirically measured to provide the static force required to move the payload. With a static coeffecient $U_{s}$ of 0.25 , one $100 \mathrm{~g}$ payload requires a force of $0.24 \mathrm{~N}$, equally a payload of $1 \mathrm{Kg}$ requires a force of $2.4 \mathrm{~N}$. On a flat surface the maximum the worm can pull is $400 \mathrm{~g}$, (a force of $0.94 \mathrm{~N}$ ), using the gearbox or the ideal waveform. Inside a confined space where all segment skins contact a surface, the maximum the worm can pull is $1.6 \mathrm{Kg}$, (a force of $3.77 \mathrm{~N}$ ), using the same two waveforms. In comparison, the sine waveform could only pull a maximum of $800 \mathrm{~g}$, (a force of $1.88 \mathrm{~N}$ ). Table II shows the range of weights used and distances achieved. As expected, the greater the weight, the less distance is achieved. The worm robot weighs a total of $225 \mathrm{~g}$ and yet the results show that it can pull a weight of $1.6 \mathrm{Kg}$, when all four segment skins are in contact. As with the previous experiment, the results of each waveform are in line with the previous results, however, the final payload of $1 \mathrm{Kg}$ was not able to be pulled by the sine waveform as it does not produce enough clamping force. These results show a dramatic performance difference between a simple sine wave control of the tendons and a purposefully designed gearbox waveform intended to achieve a longer period of segments 
TABLE II

DiSTANCE TRAVELLED THROUGH CONFINED SPACE EXECUTING 50 CYCLES.

\begin{tabular}{|c|c|c|c|}
\hline Payload (grams) & Waveform & Distance $(\mathbf{m m})$ & \% of best \\
\hline No payload & Ideal & 56 & 100.0 \\
\hline & Gearbox & 54 & 96.1 \\
\hline & Sine & 48.5 & 81.4 \\
\hline 100g payload & Ideal & 47.5 & 100.0 \\
\hline & Gearbox & 45 & 94.7 \\
\hline & Sine & 39 & 82.1 \\
\hline $\mathbf{2 0 0 g}$ payload & Ideal & 40 & 100.0 \\
\hline & Gearbox & 37.5 & 94 \\
\hline & Sine & 32.5 & 81.25 \\
\hline $\mathbf{5 0 0 g}$ payload & Ideal & 36.5 & 100.0 \\
\hline & Gearbox & 35 & 96.5 \\
\hline & Sine & 26 & 71.7 \\
\hline $\mathbf{1 K g}$ & Ideal & 31.5 & 100.0 \\
\hline & Gearbox & 30 & 96 \\
\hline & Sine & n/a & 0.0 \\
\hline
\end{tabular}

clamping.

If the results of these experiments are compared with the assumptions presented in Fig. 7, it is clear that while the sine waveform can no longer pull the payload, the results are fairly consistent. The performance drops of sine and geared are $20 \%$ and $5 \%$ respectively. This shows good confirmation that adjusting the gearbox parameters can optimise the robot worm performance to that near an ideal waveform, whilst using only one actuator.

\section{TRANSFERING MECHANISM PARAMETERS TO A GEARBOX DRIVE MECHANISM}

A state driven actuator mechanism has been designed in a soft bodied worm robot with three actuated segments. Fig. 13 shows the front view of the robot giving details of the actuator mechanism. It also shows $\mathrm{r} 1$ and $\mathrm{r} 2$, the two radii that determine the output loci shape that pulls the segment tendons. On one hand, $\mathrm{r} 1$ can be considered as the main sinusoidal carrier wave whilst $\mathrm{r} 2$ provides the offset to shape the loci.

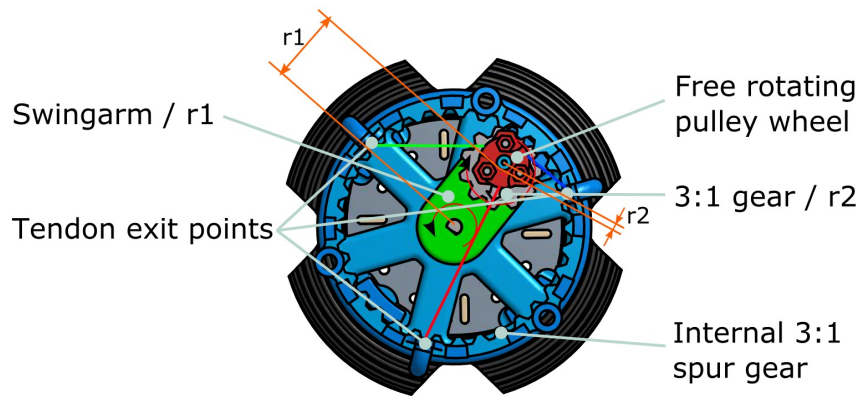

Fig. 13. Front view of worm robot showing the formation of the actuator mechanism made from two connected counter rotating gears.

Fig. 14 shows a side view of the worm robot. Each of the segments has a tendon connected through to the drive mechanism using a Bowden cable. The drive mechanism pulls each tendon to produce the gearbox waveform shown in Fig. 7 where each segment's waveform has a $120^{\circ}$ offset from its neighbour. The body of the robot has been printed on an Objet $2603 \mathrm{D}$ printer capable of printing in multiple material including rubbers. The segment walls and actuator mechanism are made from Vero white, a hard 3D printable material and the soft skin is printed using Tango plus, a soft rubber capable of deformation under influence of a tendon but with enough resistance to return to shape when the tendon forces are released. Fig. 15 shows the robot printed and assembled. Note the three separate stages each segment is in when the robot is at rest. The robot has only one actuator, a Dynamixel XL-320. The surface of each segment has a raised profile to subtly recreate setae found naturally in many annelids. Most segments of an earthworm have tiny bristle like structures called setae. These setae aid in anchoring the segment to the environment. Setae are not exclusive to earthworms and can be seen in many examples in nature, and Kim et al. [17] have shown the advantages of setae in a clamping role with a bio-mimetic inchworm robot.

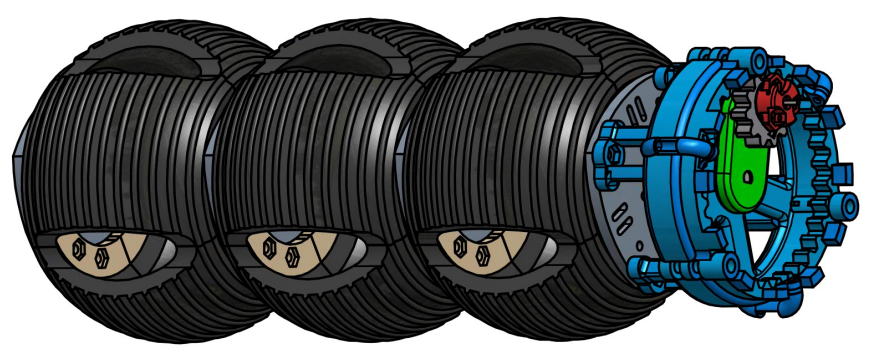

Fig. 14. Side view of robot worm design showing the front gear mechanism and soft body worm segments.

This new single motor actuated robot has been run through the same performance metric as the test platform. The performance observed was as expected to be in line with the predicted performance of the gearbox waveform. This confirms that by exploring the gearbox parameters with the initial test platform we are able to build a simplified single motor actuated peristaltic worm with comparable performance to a robot worm with additional actuators dedicated to each worm segment.

\section{CONCLUSION}

In this paper we have presented a review of the developments towards peristaltic worm locomotion and identified a lack of exploration towards simplified mechanisms using minimal actuators that are more suitable for miniaturisation. We have removed the complexity of the control signal to multiple actuators and instead embedded the control sequence within a novel drive mechanism. This drive mechanism has been implemented in to a 3D printed soft robot body with three segments. Experiments with the robot have shown successful peristaltic locomotion comparable with a robot worm that requires multiple actuators, and that a tuned waveform generated by the gearbox mechanism can achieve better locomotion distance and payload carrying capabilities 


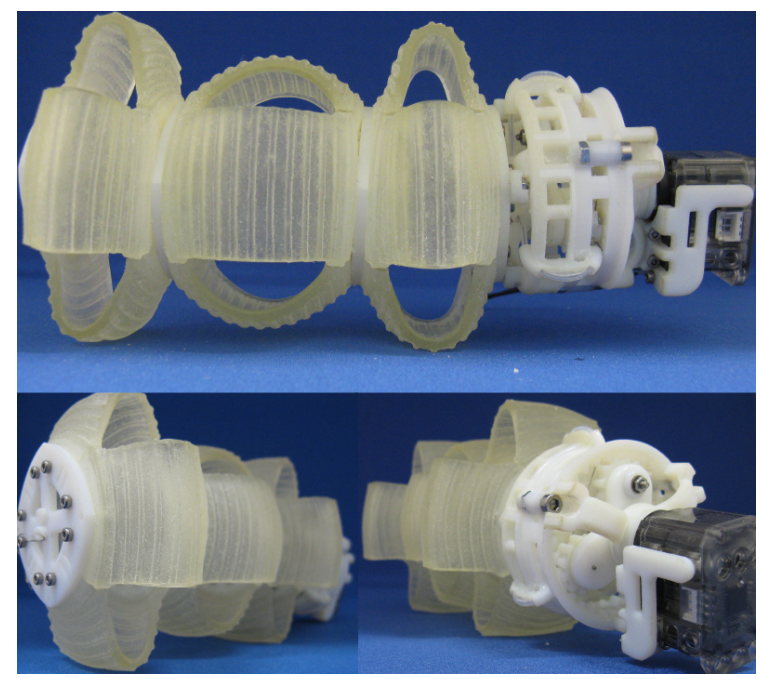

Fig. 15. Photograph of 3D printed worm robot with gearbox that drives optimised peristalatic worm locomotion.

than a simplified sine waveform. The gearbox parameters identified in this paper will enable future work to improve the robot worm to adapt to range of environment shapes.

\section{FUTURE WORK}

Two future experiments are planned with this robot. Whilst this paper has presented a method to optimise locomotion to a set environment, it has provided variables that could alter the gearbox parameters during locomotion if such a mechanism were possible. Using discrete actuators such as SMAs or Piezo Ceramic motors, small adjustments could be made to the $r_{1}: r_{2}$ ratio, or tendon offset 'a'. In doing this, the system could be compliant to varying environment dimensions. A second proposal is to integrate it with the capsule version of the TACTIP tactile sensor as presented by Winstone et al. [18]. By adding a tactile sensor to a peristaltic worm robot it provides opportunity for remote tactile sensing or palpation of difficult to reach places such as within the body. Where a difference in shape or tissue density within the GI tract indicates the presence of a tumour patients could be diagnosed earlier and more comfortably than current techniques. This integration of tactile sensing and worm locomotion would be towards an active exploration capsule endoscopy.

\section{ACKNOWLEDGEMENTS}

Bristol Robotics Laboratory gratefully acknowledge that this work was supported by the Above \& Beyond charity, http://www.aboveandbeyond.org.uk/.

\section{REFERENCES}

[1] M. A. Armada and P. de González Santos, Climbing and Walking Robots. Berlin, Heidelberg: Springer Berlin Heidelberg, 2005.

[2] B. Gramescu, C. Nitu, and N. Alexandrescu, "Modeling of a Mobile Robot with Worm-like Movement," in EUROCON 2005 - The International Conference on "Computer as a Tool", pp. 1204-1207, IEEE, 2005.
[3] A. Yamashita, K. Matsui, R. Kawanishi, T. Kaneko, T. Murakami, H. Omori, T. Nakamura, and H. Asama, "Self-localization and 3D model construction of pipe by earthworm robot equipped with omni-directional rangefinder," 2011 IEEE International Conference on Robotics and Biomimetics, ROBIO 2011, pp. 1017-1023, 2011.

[4] H. Omori, T. Murakami, H. Nagai, T. Nakamura, and T. Kubota, "Planetary subsurface explorer robot with propulsion units for peristaltic crawling," Proceedings - IEEE International Conference on Robotics and Automation, pp. 649-654, 2011.

[5] M. Quirini, S. Member, A. Menciassi, S. Scapellato, C. Stefanini, and P. Dario, "Design and Fabrication of a Motor Legged Capsule for the Active Exploration of the," IEEE/ASME TRANSACTIONS ON MECHATRONICS, vol. 13, no. 2, pp. 169-179, 2008.

[6] R. Carta, B. Lenaerts, J. Thoné, G. Tortora, P. Valdastri, A. Menciassi, R. Puers, and P. Dario, "Wireless Power Supply as Enabling Technology Towards Active Locomotion in Capsular Endoscopy," in Eurosensors, pp. 1369-1372[1] R. Carta, B. Lenaerts, J. Thoné, G. T, 2008.

[7] J. Zuo, G. Yan, and Z. Gao, "A micro creeping robot for colonoscopy based on the earthworm.," Journal of medical engineering \& technology, vol. 29, no. 1, pp. 1-7, 2005.

[8] G. Yan, D. Ye, P. Zan, K. Wang, and G. Ma, "Micro-Robot for Endoscope Based on Wireless Power Transfer," ICMA, pp. 3577-3581, aug 2007.

[9] D. Zarrouk, I. Sharf, and M. Shoham, "Conditions for worm-robot locomotion in a flexible environment: theory and experiments.," IEEE transactions on bio-medical engineering, vol. 59, pp. 1057-67, apr 2012.

[10] P. Gao, G. Yan, and Z. Wang, "A robotic endoscope based on minimally invasive locomotion and wireless techniques for human colon," ... of Medical Robotics ..., no. January, pp. 256-267, 2011.

[11] H. Omori and T. Nakamura, "Development of mobile robots based on peristaltic crawling of an earthworm," Robotics 2010: Current ..., 2010.

[12] S. Seok, C. D. Onal, K.-J. Cho, R. J. Wood, D. Rus, and S. Kim, "Meshworm: A Peristaltic Soft Robot With Antagonistic Nickel Titanium Coil Actuators,' IEEE/ASME Transactions on Mechatronics, vol. 18, pp. 1485-1497, oct 2013.

[13] A. S. Boxerbaum, K. M. Shaw, H. J. Chiel, and R. D. Quinn, "Continuous wave peristaltic motion in a robot," The International Journal of Robotics Research, vol. 31, pp. 302-318, jan 2012.

[14] A. Boxerbaum and A. Horchler, "Worms, waves and robots," Robotics and ..., pp. 3537-3538, 2012.

[15] K. a. Daltorio, A. S. Boxerbaum, A. D. Horchler, K. M. Shaw, H. J. Chiel, and R. D. Quinn, "Efficient worm-like locomotion: slip and control of soft-bodied peristaltic robots.," Bioinspiration \& biomimetics, vol. 8, no. 3, p. 035003, 2013.

[16] A. Horchler, A. Kandhari, K. Daltorio, K. Moses, K. Andersen, H. Bunnelle, J. Kershaw, W. Tavel, H. Chiel, R. Bachmann, and R. Quinn, "Worm-Like Robotic Locomotion with a Compliant Modular Mesh," in Biomimetic and Biohybrid Systems (S. P. Wilson, P. F. Verschure, A. Mura, and T. J. Prescott, eds.), vol. 9222 of Lecture Notes in Computer Science, (Barcelona), pp. 26-37, Springer International Publishing, 2015.

[17] B. Kim, M. Lee, Y. Lee, Y. Kim, and G. Lee, "An earthwormlike micro robot using shape memory alloy actuator," Sensors and Actuators A: .., vol. 125, pp. 429-437, jan 2006.

[18] B. Winstone, "Biomimetic Tactile Sensing Capsule," in ... and Biohybrid Systems, (Barcelona), pp. 113-122, Springer, 2015. 